\title{
REFERENCES
}

1. N. Dunford and J. Schwartz, Linear operators, Part II: Spectral theory, Interscience, New York, 1963.

2. P. R. Halmos, Introduction to Hilbert space, Chelsea, New York, 1957.

3. - Measure theory, Van Nostrand, New York, 1961.

4. F. J. Murray, An introduction to linear transformations in Hilbert space, Princeton Univ. Press, Princeton, N. J., 1941.

Michigan State University

\section{THE MOMENTS OF RECURRENCE TIME}

\section{J. WOLFOWITZ ${ }^{1}$}

In connection with Poincaré's recurrence theorem Kac [1] obtained the mean of the recurrence time (formula (3) below) and the author [2] gave a very simple proof of this result. Recently Blum and Rosenblatt [3] obtained ${ }^{2}$ the higher moments (formula (2) below). In the present note we obtain both results by an exceedingly simple and perspicuous argument. This note is entirely self-contained.

Let $\Omega$ be a point set, $m$ a probability measure on $\Omega$, and $T$ a one-toone ergodic measure-preserving transformation of $\Omega$ into itself. Let $A \subset \Omega$ be such that $m(A)>0$. For any point $a$ in $\Omega$ let $n(a)$ be the smallest positive integer such that $T^{n} a \in A$; if no such integer exists let $n(a)=\infty$. Define $A_{k}=\{a \in A \mid n(a)=k\}, \bar{A}=\Omega-A$, and $\Gamma_{k}$ $=\{a \in \bar{A} \mid n(a)=k\}$. Borrowing the notation of [3] we will define

$$
p_{n}=m\left\{\Gamma_{n} \cup \Gamma_{n+1} \cup \ldots\right\},
$$

for $n \geqq 1$. We will also make use of the usual combinatorial symbol $(k)_{j}=k(k-1) \cdots(k-j+1)$ for $k$ and $j$ posilive integers, with $(k)_{0}=1$.

Our object will be to prove that

$$
D_{j}=\int_{A}[n(a)]_{j} d m=j(j-1) \sum_{k=j-2}^{\infty}(k)_{(j-2)} p_{k+1}
$$

Received by the editors August 1, 1966.

1 Fellow of the John Simon Guggenheim Memorial Foundation. Research supported in part by the U. S. Air Force under Contract AF 18(600)-685 with Cornell University.

2 These moments have also been obtained by F. H. Simons, Notice \#40 of the Eindhoven Technical School, December 23, 1966. 
for $j \geqq 2$, the result of [3]. The result of [1] (also proved in [2]) is

$$
D_{1}=1 \text {. }
$$

By Poincaré's recurrence theorem (e.g., [2]; ergodicity of $T$ is not required) one has that $m\left(A_{\infty}\right)=0$. The ergodicity of $T$ implies that $m\left(\Gamma_{\infty}\right)=0$.

The basic formula of our argument will be

$$
T\left(A_{k} \cup \Gamma_{k}\right)=\Gamma_{k-1}
$$

for $k \geqq 2$; it is so obvious as not to require proof. Using (4) repeatedly for $k=n+1, n+2, \cdots$ we obtain that

$$
\begin{aligned}
& m\left(\Gamma_{n}\right)=\sum_{k=n+1}^{\infty} m\left(A_{k}\right), \quad n \geqq 1 ; \\
& p_{n}=\sum_{k=n+1}^{\infty}(k-n) m\left(A_{k}\right), \quad n \geqq 1 .
\end{aligned}
$$

Thus

$$
\begin{aligned}
p_{1} & =m\left(A_{2}\right)+2 m\left(A_{3}\right)+3 m\left(A_{4}\right)+\cdots \\
& =D_{1}-\sum_{k=1}^{\infty} m\left(A_{k}\right)=D_{1}-m(A) .
\end{aligned}
$$

Obviously

$$
p_{1}=m(\bar{A})=1-m(A),
$$

so that (7) and (8) prove (3).

Using (6) in the right member of (2) we obtain that the coefficient of $m\left(A_{k}\right), k \geqq j$, in the right member of (2) is

$$
j(j-1)\left[\sum_{i=1}^{k-j+1} i(k-i-1)_{(j-2)}\right],
$$

which is easily shown (e.g., by induction) to equal $(k)_{j}$. This proves (2).

\section{REFERENCES}

1. M. Kac, On the notion of recurrence in discrete stochastic processes, Bull. Amer. Math. Soc. 53 (1947), 1002-1010.

2. J. Wolfowitz, Remarks on the notion of recurrence, Bull. Amer. Math. Soc. 55 (1949), 394-395.

3. J. R. Blum and J. I. Rosenblatt, On the moments of recurrence time, University of New Mexico mimeographed report, 1966; J. Math. Sci. (Delhi) 2 (1967), 1-6.

Cornell University 